\title{
The study of a risk model based on martingale theory
}

\author{
Xiaoqing Yun ${ }^{1, \text { a }}$ \\ ${ }^{1}$ Liren College of Yanshan University, Qinhuangdao China \\ ayunxq@ysu.edu.cn
}

\begin{abstract}
Due to the classical risk model has many hypotheses which cause limitation, the two-insurance double Poisson risk model is set up, when the two-insurance claim follow exponential distribution, and one insurance claim follow exponential distribution, the other insurance claim follow combination of exponential distributions, specific ruin probability formula are got.
\end{abstract}

Keywords: Ruin probability; Exponential distribution; Combination of exponential distributions.

\section{Introduction}

As one of the main research objects in risk theory, a lot of research about ruin probability has been carried on by some scholars. In 1903, the Swedish actuary Fillip Lundberg initiated the study of ruin theory, later, the Swedish school headed by Harald Cramer improved the ruin theory of Fillip Lundberg, so the study of ruin theory has a solid foundation of mathematics. The exact expressions, relevant assumptions and the main results of the classical ruin model were given by them. The ruin theory of Lundberg and Cramer has been the basic theory of the classical ruin theory.

In the classical risk model [1.2] $U(t)=u+c t-\sum_{k=1}^{N(t)} X_{k}, t \geq 0, u$ is initial reserve, $C$ denote premium rate per unit time, $X_{k}$ counts the kth claim amount, $N(t)$ obey the Poisson distribution, and $N(t)$ denote the claim number in the interval $(0, t] .\left\{X_{k} k \geq 1\right\}$ are nonnegative sequence of independent and identically distributed random variables, $N(t)$ and $\left\{X_{k} k \geq 1\right\}$ are independent 
of each other. Because the classical risk model has many hypotheses which cause limitation, many scholars generalized the classical risk model. The classical risk model and the expanded model in the work [3.4] describe risk management process of single insurance. With the expansion of business scale of the insurance company, the study of multiple line risk model has much breakthrough, in the work [5], a class of multiple line risk model is constructed, the ruin probability is gotten, the work [6] study the two-insurance Poisson risk model and its ruin probability, in these works, premium income is constant in unit time, in fact, premium income process is random process, in the work [7], though the premium income process is generalized to Poisson process, claim process is management process of single insurance. In this paper, considering two-insurance, the double Poisson risk model in which premium income process and claim process are Poisson process is constructed, the ruin probability and relevant formula are gotten with martingale theory, at last, when the two-insurance claim follow exponential distribution, and one insurance claim follow exponential distribution, the other insurance claim follow combination of exponential distributions, specific ruin probability formulae are got.

\section{Model description}

Definition1. Given the probability space $(\Omega, F, P)$, Assume $u \geq 0, t \geq 0$, the two-insurance double Poisson risk model is

$$
R(t)=u+k M(t)-\sum_{i=1}^{N_{1}(t)} X_{i}^{1}-\sum_{i=1}^{N_{2}(t)} X_{i}^{2} .
$$

In the model, $u$ is the initial reserve of insurance company, $k$ is the insurance premium of per policy. $M(t)$ denote policy total number in the interval $(0, t]$, and $M(t)$ follow Poisson distribution with parameter $\alpha \cdot N_{k}(t),(k=1,2)$ are Poisson process with intensity $\beta_{i}(i=1,2), N_{k}(t),(k=1,2)$ denote two-insurance claim arrive process, $N_{1}(t)$ and $N_{2}(t)$ are independent of each other. $X_{i}^{1}$ are nonnegative sequence of independent and identically distributed random variables, their distribution function is $F_{1}(x), E\left(X_{i}^{1}\right)=u_{1}, X_{i}^{2}$ are nonnegative sequence of independent and identically distributed random variables, their distribution function is $F_{2}(x), E\left(X_{i}^{2}\right)=u_{2} \cdot R(t)$ denote the surplus of 
insurance company at time t, $S(t)=k M(t)-\sum_{i=1}^{N_{1}(t)} X_{i}^{1}-\sum_{i=1}^{N_{2}(t)} X_{i}^{2}$ denote profit process of insurance company at time t.

\section{Lemma}

Lemma1[8]. Profit process $\{S(t) ; t \geq 0\}$ has these properties (1) $S(0)=0$ (2) process with independent increments and stationary (3) $E[S(t)]=\left(k \alpha-\beta_{1} u_{1}-\beta_{2} u_{2}\right) t \quad$ (4) there exists positive number $r$,makiing $E\left[e^{-r s(t)}\right]<\infty$.

Lemma2[9]. Assume $T$ is the ruin time, based on martingale theory, the ruin probability of the two-insurance double Poisson risk model is

$$
\psi(u)=\frac{e^{-R u}}{E\{\exp [-R R(T) \mid T<\infty]\}} .
$$

\section{Main Results}

\section{Theorem 1.The}

function $g(r)=\alpha\left(e^{-r k}-1\right)+\beta_{1}\left[M_{X_{i}^{1}}(r)-1\right]+\beta_{2}\left[M_{X_{i}^{2}}(r)-1\right]$ exist, for the profit process $\{S(t) ; t \geq 0\}$, such that $E\left[e^{-r s(t)}\right]=e^{\operatorname{tg}(r)}$.

Where $M_{X_{i}^{k}}(r)=E\left(e^{r X_{i}^{k}}\right), k=1,2$.

$$
\begin{gathered}
\text { Proof: } E\left[e^{-r s(t)}\right]=E\left\{\exp (-r)\left[k M(t)-\sum_{i=1}^{N_{1}(t)} X_{i}^{1}-\sum_{i=1}^{N_{2}(t)} X_{i}^{2}\right]\right\} \\
=E[\exp (-r) k M(t)] E\left[\exp r \sum_{i=1}^{N_{1}(t)} X_{i}^{1}\right] E\left[\exp r \sum_{i=1}^{N_{2}(t)} X_{i}^{2}\right] \\
=\exp \left[\alpha t\left(e^{-r k}-1\right)\right] \exp \left[\beta_{1} t\left(M_{X_{i}^{1}}(r)-1\right)\right] \\
\exp \left[\beta_{2} t\left(M_{X_{i}^{2}}(r)-1\right)\right] \\
=\exp \left\{\alpha\left(e^{-r k}-1\right)+\beta_{1}\left[M_{X_{i}^{1}}(r)-1\right]+\beta_{2} M_{X_{i}^{2}}(r)-1\right\} t .
\end{gathered}
$$


Theorem 2. The equation $g(r)=0$ has unique positive solution $R$, which is called as adjustment coefficient.

Proof: $g^{\prime}(r)=-\alpha k e^{-r k}+\beta_{1} E\left(X_{i}^{1} e^{X_{i}^{1}}\right)+\beta_{2} E\left(X_{i}^{2} e^{r X_{i}^{2}}\right)$,

$$
g^{\prime \prime}(r)=\alpha k^{2} e^{-r k}+\beta_{1} E\left[\left(X_{i}^{1}\right)^{2} e^{r X_{i}^{1}}+\beta_{2} E\left[\left(X_{i}^{2}\right)^{2} e^{r X_{i}^{2}}\right]>0 .\right.
$$

Because $g^{\prime \prime}(r)>0$, so $g^{\prime}(r)$ is monotone increasing in the interval $[0,+\infty), g(r)$ is convex downward shape in the interval $[0,+\infty)$, when $r \rightarrow+\infty, g^{\prime}(r) \rightarrow+\infty$. Due to $g^{\prime}(0)=-\alpha k+\beta_{1} \mu_{1}+\beta_{2} \mu_{2}<0$, so $r^{*} \in(0,+\infty)$ exists, makes $g^{\prime}\left(r^{*}\right)=0$.According to sufficient condition of extreme value, $g\left(r^{*}\right)$ is minimum value in the interval $[0,+\infty)$. Because of $g(0)=0$, when $r \rightarrow+\infty, g(r) \rightarrow+\infty$,so $g(r)$ is monotone decreasing in the interval $\left(0, r^{*}\right)$, is monotone increasing in the interval $\left(r^{*},+\infty\right)$,at last $g(r)=0$ has unique positive solution $R \in\left(r^{*},+\infty\right)$.

Theorem 3. In the two-insurance double Poisson risk model, if the claim sizes $X_{i}^{1}, X_{i}^{2}$ are independent of each other, and respectively follow exponential d i s t r i b u t i o n w i t h p a $\mathrm{r}$ a $\mathrm{m}$ e $\mathrm{t}$ e $\mathrm{r}$ $\lambda_{1}>0, \lambda_{2}>0$, then $\Psi(u)=\frac{\lambda_{2}-R}{\lambda_{2}} e^{-R u}$, where $\lambda_{1}>\lambda_{2}, R$ is adjustment coefficient.

Proof: suppose $Z=X_{i}^{1}+X_{i}^{2}, f_{z}(z)$ is probability density function of $Z$, then

$$
\begin{gathered}
f_{z}(z)=\int_{-\infty}^{+\infty} f_{X_{i}^{1}}(x) f_{X_{i}^{2}}(z-x) d x=\int_{0}^{+\infty} \lambda_{1} e^{-\lambda_{1} x} \lambda_{2} e^{-\lambda_{2}(z-x)} d x= \\
\int_{0}^{+\infty} \lambda_{1} e^{-\lambda_{1} x} \lambda_{2} e^{-\lambda_{2}(z-x)} d x=\lambda_{1} \lambda_{2} e^{-\lambda_{2} z} \int_{0}^{+\infty} e^{\left(\lambda_{2}-\lambda_{1}\right) x} d x= \\
\frac{\lambda_{1} \lambda_{2}}{\lambda_{1}-\lambda_{2}} e^{-\lambda_{2} z} \quad\left(\lambda_{1}>\lambda_{2}\right) .
\end{gathered}
$$

Surplus of insurance company before the ruin time $T$ is denoted as $\bar{R}$, then $-R(T)>x$ and $\left\{X_{i}^{1}+X_{i}^{2}>\bar{R}+x \mid X_{i}^{1}+X_{i}^{2}>\bar{R}\right\}$ are equivalent, where $X_{i}^{1}+X_{i}^{2}$ is claim amount causing ruin, so we have 


$$
\begin{gathered}
P\{-R(T)>x \mid T<\infty\}=P\left\{X_{i}^{1}+X_{i}^{2}>\bar{R}+x \mid X_{i}^{1}+X_{i}^{2}>\bar{R}\right\}= \\
\frac{P\left\{X_{i}^{1}+X_{i}^{2}>\bar{R}+x, X_{i}^{1}+X_{i}^{2}>\bar{R}\right\}}{P\left\{X_{i}^{1}+X_{i}^{2}>\bar{R}\right\}}=\frac{\int_{\bar{R}+x}^{+\infty} \frac{\lambda_{1} \lambda_{2}}{\lambda_{1}-\lambda_{2}} e^{-\lambda_{2} z} d z}{\int_{\bar{R}}^{+\infty} \frac{\lambda_{1} \lambda_{2}}{\lambda_{1}-\lambda_{2}} e^{-\lambda_{2} z} d z}=e^{-\lambda_{2} x}
\end{gathered}
$$

Furthermore, we have $\frac{d}{d x} P\{-R(T)<x \mid T<\infty\}=\lambda_{2} e^{-\lambda_{2} x}$,

$\int_{0}^{+\infty} e^{R x}$ $E\{\exp [-R R(T) \mid T<\infty]\}=$ $\psi(u)=\frac{e^{-R u}}{E\{\exp [-R R(T) \mid T<\infty]\}}=\frac{\lambda_{2}-R}{\lambda_{2}} e^{-R u}$.

Theorem 4. In the two-insurance double Poisson risk model, if the claim sizes $X_{i}^{1}, X_{i}^{2}$ are independent of each other, and $X_{i}^{1}$ follow exponential d i s t r i b u t i o n w i t h p a r a m e t e r $\lambda>0, X_{i}^{2}$ follow mixed exponential distribution $p a e^{-a y}+q b e^{-b y}$, w $\quad \mathrm{h} \quad$ e $\quad$ r $\quad$ e $\quad p+q=1, p>0, q>0$ $a>0, b>0$ then $\psi(u)=\frac{\frac{p \lambda}{\lambda-a} e^{-a \bar{R}}+\frac{q \lambda}{\lambda-b} e^{-b \bar{R}}}{\frac{p a \lambda}{(\lambda-a)(a-R)} e^{-a \bar{R}}+\frac{q b \lambda}{(\lambda-b)(b-R)} e^{-b \bar{R}}} e^{-R u}, R$ is positive $\begin{array}{llllllllll}\mathrm{s} & \mathrm{o} & \mathrm{l} & \mathrm{u} & \mathrm{t} & \mathrm{i} & \mathrm{o} & \mathrm{n} & \mathrm{o} & \mathrm{f}\end{array}$ $\alpha\left(e^{-r c}-1\right)+\beta_{1}\left(\frac{\lambda}{\lambda-r}-1\right)+\beta_{2}\left(\frac{p a}{a-r}+\frac{q b}{b-r}-1\right)=0$.

Proof: suppose $Z=X_{i}^{1}+X_{i}^{2}, f_{z}(z)$ is probability density function of $Z$, then 


$$
\begin{aligned}
& f_{z}(z)=\int_{-\infty}^{+\infty} f_{X_{i}^{1}}(x) f_{X_{i}^{2}}(z-x) d x= \\
& \int_{0}^{+\infty} \lambda e^{-\lambda x}\left[p a e^{-a(z-x)}+q b e^{-b(z-x)}\right] d x=p \frac{\lambda a}{\lambda-a} e^{-a z}+q \frac{\lambda b}{\lambda-b} e^{-b z},
\end{aligned}
$$

where $\lambda>a, \lambda>b$,furthermore

$$
\begin{aligned}
& P\{-R(T)>x \mid T<\infty\}=\frac{\int_{\bar{R}+x}^{+\infty} p \frac{\lambda a}{\lambda-a} e^{-a z}+q \frac{\lambda b}{\lambda-b} e^{-b z} d z}{\int_{\bar{R}}^{+\infty} p \frac{\lambda a}{\lambda-a} e^{-a z}+q \frac{\lambda b}{\lambda-b} e^{-b z} d z}= \\
& \frac{\frac{p \lambda}{\lambda-a} e^{-a(\bar{R}+x)}+\frac{q \lambda}{\lambda-b} e^{-b(\bar{R}+x)}}{\frac{p \lambda}{(\lambda-a)} e^{-a \bar{R}}+\frac{q \lambda}{(\lambda-b)} e^{-b \bar{R}}}
\end{aligned}
$$

$$
\frac{d}{d x} P\{-R(T)<x \mid T<\infty\}=\frac{d}{d x}\left[1-\frac{\frac{p \lambda}{\lambda-a} e^{-a(\bar{R}+x)}+\frac{q \lambda}{\lambda-b} e^{-b(\bar{R}+x)}}{\frac{p \lambda}{(\lambda-a)} e^{-a \bar{R}}+\frac{q \lambda}{(\lambda-b)} e^{-b \bar{R}}}\right]=
$$

$$
\frac{\frac{p a \lambda}{\lambda-a} e^{-a(\bar{R}+x)}+\frac{q b \lambda}{\lambda-b} e^{-b(\bar{R}+x)}}{\frac{p \lambda}{(\lambda-a)} e^{-a \bar{R}}+\frac{q \lambda}{(\lambda-b)} e^{-b \bar{R}}}
$$

so we get

$E\{\exp [-R R(T) \mid T<\infty]\}=$

$\int_{0}^{+\infty} e^{R x} \frac{\frac{p a \lambda}{\lambda-a} e^{-a(\bar{R}+x)}+\frac{q b \lambda}{\lambda-b} e^{-b(\bar{R}+x)}}{\frac{p \lambda}{(\lambda-a)} e^{-a \bar{R}}+\frac{q \lambda}{(\lambda-b)} e^{-b \bar{R}}} d x=$ 


$$
\begin{aligned}
& \frac{\frac{p a \lambda}{(\lambda-a)(a-R)} e^{-a \bar{R}}+\frac{q b \lambda}{(\lambda-b)(b-R)} e^{-b \bar{R}}}{\frac{p \lambda}{\lambda-a} e^{-a \bar{R}}+\frac{q \lambda}{\lambda-b} e^{-b \bar{R}}} \text {, and } \\
& \psi(u)=\frac{\frac{p \lambda}{\lambda-a} e^{-a \bar{R}}+\frac{q \lambda}{\lambda-b} e^{-b \bar{R}}}{\frac{p a \lambda}{(\lambda-a)(a-R)} e^{-a \bar{R}}+\frac{q b \lambda}{(\lambda-b)(b-R)} e^{-b \bar{R}}} e^{-R u} .
\end{aligned}
$$

From

let $g(r)=\alpha\left(e^{-r k}-1\right)+\beta_{1}\left[M_{X_{i}^{1}}(r)-1\right]+\beta_{2}\left[M_{X_{i}^{2}}(r)-1\right]=0$, because

$$
\begin{aligned}
& M_{X_{i}^{1}}(r)=\frac{\lambda}{\lambda-r}, \\
& M_{X_{i}^{2}}(r)=E\left(e^{r X_{i}^{2}}\right)=\int_{0}^{+\infty} e^{r x}\left(p a e^{-a x}+q b e^{-b x}\right) d x=\frac{p a}{a-r}+\frac{q b}{b-r},
\end{aligned}
$$

so $R$ is positive solution of

$$
g(r)=\alpha\left(e^{-r k}-1\right)+\beta_{1}\left(\frac{\lambda}{\lambda-r}-1\right)+\beta_{2}\left(\frac{p a}{a-r}+\frac{q b}{b-r}-1\right)=0 .
$$

\section{Conclusions}

This paper studies the double Poisson risk model. When the two-insurance claim follow exponential distribution and combination of exponential distributions, we derive the corresponding explicit ruin probability formula. To the risk model, proving method is strict, conclusions are explicit.

\section{Acknowledgment}

This work is supported by Qinhuangdao foundation (201302A221).

\section{References}

[1] H. U. Gerber, Mathematical fun with ruin theory, Insurance: Mathematics and Economics, vol.7, no.1, pp.15-23, 1988

[2] Cheng Shixue, The survey for researches of ruin theory, Advances in 
Mathematics, vol.31, no.5, pp.403-422, 2002

[3] YANG Shan-chao, Approximation of ruin probability $\psi(0)$ in the compound binomial model, Journal of guangxi normal university, vol.21, no.4, pp.48-52, 2003

[4] DONG Hua, LIU Zai-ming and ZHAO Xiang-hua, Ruin problem for a class of risk model with random income, Acta mathematicae applicatae sinica, vol.34, no.4, pp.683-695, 2011

[5] YU Wen-guang, ZHANG Chun-ming and LI Ai-qin, Creation and ruin theory of the time surplus process of the multi-risk model with stochastic interest rates, Journal of hefei university of technology, vol.32, no.9, pp.1445-1448, 2009

[6] YU Wan-xia and WANG Han-xing, Poisson risk model for two-type-risk insurance and the ruin probability, Journal of shanghai university(natural science), vol.9, no.6, pp.529-532, 2003

[7] Hua Dong,Zaiming LIU, The ruin problem in a renewal risk model with two-sided jumps, Mathematical and computer modelling, pp.800-811, (57)2013

[8] GRANDEL J, Aspect of risk theory[M], New York:Spring-Verlag,1991

[9] GONG Ri-chao and LI Feng-jun, Ruin probability in risk model with two Poisson processes, Journal of xiangtan nomal university (natural science edition), vol.23, no.1, pp.55-57, 2001 